\title{
The Future of the Use of Herbal Medicines in the Treatment of Cancer
}

\author{
Wagner Loyola* \\ Laboratory of Health and Genetics, Concordia/Santa Catarina State, Brazil
}

*Corresponding author: Wagner Loyola, Laboratory of Health and Genetics, Concordia/Santa Catarina State, Brazil.

Received Date: November 14, 2018

Published Date: December 14, 2018

\begin{abstract}
Modern life puts humans in contact with various carcinogenic agents. The incidence of tumors in the world's population has grown significantly in the 2000s. Lung tumors are one of the leading causes of death in the world WHO[1], and they are noncommunicable diseases. Despite significant increase in cancer related mortality, lack of efforts to treat and prevent cancer especially in underdeveloped countries. The use of chemotherapy and radiotherapy is still a necessity in the treatment of neoplasms. However, these strategies have adverse effects that increase the morbidity of the cases. Another advantage in the use of substances of vegetal origin is its low toxicity, with side effects being minimal. Plant proteins by their adjuvant action may exert a synergistic action with the traditional treatments, improving the prognosis of the treatment of tumors.
\end{abstract}

Keywords: Adjuvant; Immunology; Cancer; Phytotherapy; Vaccine

\section{Introduction}

Modern life puts humans in contact with various carcinogenic agents. The incidence of tumors inthe world's population has grown significantly in the 2000s. Lung tumors are one of the leading causes of death in the world WHO [1], and they are no communicable diseases. The same increase was not observing in the attempt of treatment or prevention, especially in underdeveloped countries.In the treatment of tumors, certainly early diagnosis is the best form of treatment. However, this does not occur, especially with poor populations. The need for research into forms of treatment becomes an important global health issue. The research of forms of treatment to prevent or at least reduce the number of cases, would justify the cost of this process. The main strategy for the prevention of various pathogens is the production of vaccines. This immunobiological is very effective and prevention is always the best way to treat any type of disease, be it a tumor or infection. However, the production of an effective vaccine is a time-consuming and expensive process, which makes it difficult to use on a large scale.The main function of the vaccine is the activation of lymphocytes, capable of recognizing and eliminating tumor cells. The production of memory cells will give the individual a lasting protection against the formation of neoplastic masses. For this, it is necessary to identify molecules that serve as antigen and stimulate the immune system.

Another important factor in the production of a vaccine is the addition of adjuvant. These substances serve to amplify the action triggered by the antigen in the activation of the immune system. This increased response is summarizing in increased antigen presentation molecules and cytokine production that will modulate the immune response. This set of actions will ensure an efficient and protective response.Various substances are using as adjuvants, from metal salts (aluminum carbonates) to saponins of vegetable origin. The importance of adjuvants increases as the understanding of cooperation between innate and adaptive immune response increases. While lymphocytes recognize a single molecule in nature, innate immune response cells recognize molecular patterns through a family of receptors called pattern recognition receptors (PRRs). Within this family, we find several receptors that can identify from bacterial LPS, to cell wall carbohydrates from fungi.

PRRs were for many years related only to non-specific antigen recognition. However, with the advancement of the study on cytoplasmic signaling pathways of the innate immune response, it now knows that modulation through the production of cytokines from these cells is an important factor for the development of a protective immune response. The most studied are mannose (MR) receptors, beta-glucan receptors (Dectin) and Toll-like receptors (TLRs). The latter is composed of a family of nine receptors present mainly in macrophages, neutrophils and mature dendritic cells. Various substances of plant origin (herbal medicines) can be recognizing by PRRs. These substances may be present in seeds, roots, stems or leaves. Although it does not confer a specific response, 
activation of cytoplasmic signaling pathways in innate response cells leads to secretion of cytokines that will modulate lymphocyte response. Among the important ways to combat cancer, we can mention Nf-kB and MyD88. Signaling through these two pathways is responsible for the secretion of cytokines, which will stimulate the development of CD4 + T lymphocytes, which differentiate into Th1 lymphocytes. The Th1 profile is responsible for the secretion of cytokines that will assist cytotoxic and phagocytic cells, both in cell multiplication and in increasing tumoricidal capacity.Combating tumors and infectious diseases should occur in the shortest possible time. In this criterion, early signaling plays a key role in preventing the development of tumors. Several proteins of plant origin have demonstrated this ability when recognized by the PRR, present on the surface of cells of the innate response. Among the several proteins studied, a protein called Artin-M, purified from seeds of Artocarpus integrifolia, has shown a promising action. This protein is recognizing by the CXCR2 receptor present on the surface of neutrophils Loyola et al.[2]. Artin M induced the production of IL$12 \mathrm{p} 40$ and IFN- $\gamma$ in vivo and stimulated a protective effect against Leishmania major infection. These cytokines are also important for the control of tumors, since they induce proliferation, adhesion and increase of tumoricidal capacity. This early action is critical for decreasing the proliferation of tumor cells.

Another protein that demonstrates protective action in the fight against neoplasia is concanavalin A. Protein extracted from the seed of Canavalia ensiformis; at first, the lymphoproliferative action was confirming. Further studies with this lectin have demonstrated immunomodulatory action through binding to Toll Like Receptors 4 (TRL4) present on the surface of macrophages and neutrophils. The binding triggers signaling that induces IL-12 and IFN- $\gamma$ cytokine secretion, inducing a strong Th1 response. Loyola et al. [3] demonstrated chemotactic action for neutrophils, which associated with activated macrophages, protected mice experimentally infected with Candida albicans.Saponins extracted from Glycine max ODA et al. [4], Pulsatilla chinensis Sun et al.[5], Polygala Senega L Estrada et al. [6] have demonstrated immunomodulatory action. This action is characterizing mainly by the secretion of cytokines that influence the development of the lymphocyte profile, thus demonstrating an influence of the innate immune response on the development of the adaptive response. In addition to the pleiotropic action of these cytokines, they may increase the production capacity of oxidative reagents by cells of the innate immune response.

Through ethnobotany (branch of botany that studies the use of plants by peoples), we can select several plant species producing new substances with antineoplastic action. In this article, I focused only on adjuvant action, since research for cancer vaccines is the goal of several research groups. We could also mention that several plant species may exert a direct tumoricidal action, but the direct action of herbal medicines has not been showing to be effective, especially in advanced stage tumors such as Equinacea purpurea, a plant of American Indian medicine Brush et al. [7]. Nevertheless, when associated with traditional therapies, they demonstrate satisfactory results.The use of chemotherapy and radiotherapy is still a necessity in the treatment of neoplasms. However, these strategies have adverse effects that increase the morbidity of the cases. Another advantage in the use of substances of vegetal origin is its low toxicity Conchon-Costa et al. [8], with side effects being minimal. Plant proteins by their adjuvant action may exert a synergistic action with the traditional treatments, improving the prognosis of the treatment of tumors.

\section{Conclusion}

Future studies may demonstrate the role of PRRs in the activation of tumoricidal mechanisms. These mechanisms will have very early activation, which will improve the prognosis for the patient with cancer. However, each receptor binds to a substance present in plant extracts. The vast majority of studies use extracts, which represent a set of substances. For the use in the treatment of neoplasia, it will be necessary the isolation and purification of these substances protagonists in the anticancer response.The identification of receptors and their plant agonists may emerge as new drugs in the treatment of tumors. Perhaps, it is not the cure, since there are several types of tumors and each with its characteristic. Nevertheless, it would be plausible to say that the advances in these studies may confer an improvement in prognosis and in the life span of patients with tumors.

\section{Acknowledgment}

None.

\section{Conflict of Interest}

No conflict of interest.

\section{References}

1. World Health Organization (2016).

2. Loyola W, Custódio LA, Felipe I, Conchon-Costa I, Carvalho PG, et al. (2012) Artin M enhances TNF- $\alpha$ production and phagocytosis of Candida albicans mediated by dectin-1 and mannose receptors. Int Immunopharmacol 12(2): 378-383.

3. Loyola W, Gaziri DA, Gaziri LC, Felipe I (2002) Concanavalin A enhances phagocytosis and killing of Candida albicans by mice peritoneal neutrophils and macrophages. FEMS Immunol Med Microbiol 33(3): 201-208.

4. Oda K, Matsuda H, Murakami T, Katayama, Ohgitani T, et al. (2003) Relationship between adjuvant activity and amphipathic structure of soyasaponins. Vaccine 21(17-18): 2145-2151.

5. Sun Y, Liu J, Yu H, Gong C (2010) Isolation and evaluation of immunological adjuvant activities of saponins from the roots of Pulsatilla chinensis with less adverse reactions. Int Immunopharmacol 10(5): 584-590.

6. Estrada A, Katselis GS, Laarvelda B, Barl B (2000) Isolation and evaluation of immunological adjuvant activities of saponins from Polygala senega $\mathrm{L}$. Comparative Immunology, Microbiology and Infectious Diseases. 23(1): 27-43.

7. Brush J, Mendenhall E, Guggenheim A, Chan T, Connelly E, et al. (2006) The effect of Echinacea purpurea, Astragalus membranaceus and Glycyrrhiza glabra on CD69 expression and immune cell activation in humans. Phytother. Res 20(8): 687-695.

8. Conchon-Costa I, Loyola W, Gaziri LCJ, Custódio LA, Felipe I (2007) Low dose of concanavalin A enhances innate immune response and prevents liver injury in mice infected with Candida albicans. FEMS Immunol Med Microbiol 49: 330-336. 